\title{
STRUCTURAL PROPERTIES AND OPERATIONAL PHILOSOPHY OF THE VEHICLE WITH THE QUANTUM ENGINE
}

\author{
Jurij Kotikov \\ Saint Petersburg State University of Architecture and Civil Engineering \\ Vtoraja Krasnoarmejskaja ul. 4, St. Petersburg, Russia \\ cotikov@mail.com
}

\begin{abstract}
Successes of physics of last decades have designated prospect of the new technological way; use of antigravitational principles of moving of objects and a propulsive drive will be its basis. At conceptual level possibilities of use of the quantum engine (QuEn) on motor transport are considered.

QuEn does not demand the external energy carrier on the basic operating modes of the car. QuEn, creating impulse of the traction force transferred directly on the frame of a vehicle, does a power drive of wheels unnecessary - all the wheels work in a conducted mode.

Formation of vertical making traction force can provide partial suspension or pressing down car that is important for flotation ability. A number of other properties is mentioned also. Attempt of forecasting of configuration of the QuEn-car is carried out.
\end{abstract}

\footnotetext{
Keywords

Automobile, the quantum engine, structural properties, operational philosophy.
}

\section{Introduction}

By the beginning of the 21st century, physics advances have brought the humanity to the era of quantum energetics using global vacuum energy. Breakthrough theories of $B$. Parker (Parker, 1991), P. Davies (Davies, 1985), H. Puthoff (Puthoff, 2010), A. Veinik (Veinik, 1991), A. Nikitin (Nikitin, 2016), V. Leonov (Leonov, 1996, 1997, 2002, 2009, 2010, 2013, 2016, 2017a, 2017b, 2017c, 2017d) and other physicists were somehow aimed at searching, discovering and formalizing of the unified field, the existence of which was predicted by Einstein (Parker, 1991).

Unveiling the unified field comprises the key to mastering the ability to draw energy from the world's vacuum.

Realization of this phenomenon will result in a new technological paradigm involving the transport sector as well. And then, quantum engines will likely replace internal combustion and jet engines. Let us briefly analyze what structural and operational properties of vehicles with the quantum engine will be affected by those changes.

At first, it is necessary to review physical foundations of quantum energetics.
Physics advances at the turn of the 20th and 21st centuries

Modern energetics is based on the fundamental knowledge gained in the 19th and 20th centuries: the Faraday's law of induction, Maxwell's equations, discoveries of the kinetic-molecular theory of heat, quantum of radiation, structure of the atomic nucleus, radioactivity, etc. Those discoveries made it possible to use the energy of chemical fuel and energy of the atomic nucleus to produce mechanical and thermal energy, perform their conversion into electrical energy and deliver such energy to the consumer (Kotikov, 2006). However, the latest achievements in physics ((Leonov, 2017a) and others) allow coming ever closer to the establishment of a whole new level of energetics - quantum energetics which will form the basis for a new technological paradigm in the world economy.

Applied physics of the 20th century considered the space-time as absolute emptiness with no structure (based on verified and utilized practical effects and achievements in theoretical physics of the past decades) (Leonov, 2017b). 
Einstein introduced the concept of the unified field (replacing the mechanistic ether having no experimental evidences with the concept of four-dimensional spacetime as the unified field (Shkrudnev, 2017)), but he did not consider space as absolute emptiness. He believed that "the existence of an empty space must be excluded" (Parker, 1991).

Einstein dreamed of a unified theory which would cover all physical phenomena - the unified field theory. But he never succeeded in creation of such theory covering all physical phenomena and overcoming the gap between the general theory of relativity and the quantum theory, giving a unified interpretation of all fields and their interaction with elementary particles (although he devoted the last 30 years of his life to this). Other major scientists - Heisenberg, Eddington, Pauli (Pauli, 1975) - also dedicated decades of their lives to achieving this goal which was unattainable for them. This can be explained by the fact that at that time there were not enough developments in theoretical and experimental physics to formalize their insights.

The Einstein's idea of the space-time field structure as a unified field (Parker, 1991), the Davies's idea of "superforce" uniting everything in the universe (Davies, 1985 ) and the Puthoff's idea of great energy density of the physical vacuum (Puthoff, 2010) were taken together and implemented by Russian scientist Leonov V. S. in his theory of the elastic quantized medium (EQM) (Leonov, 1996, 1997) and the theory of Superunification after discovery of the space-time quantum (quanton) (Leonov, 2010a).

The EQM theory determines the structure of the vacuum space as densely filled with the zero element (ZE) of the Mendeleev's periodic table (Leonov, 2017c). Mendeleev predicted its existence, tried to find it and even included it into the original version of the periodic table. He named it newtonium (Mendeleev, 1905). Mendeleev put high hopes on this imperceptible element. "Neither gravity nor any of the problems of energy can be rightly understood without a real conception of the ether as a universal medium transmitting energy at a distance", he wrote (Mendeleev, 1905). However, since neither the author of the periodic table nor any of his contemporaries were able to determine, formalize or prove the existence of the zero element, this hypothetical element was removed from the periodic table adopted by the scientific community for practical use.

The zero element missing in the periodic table (according to Mendeleev, the remaining elements cannot be formed without it) was brought back to life by Leonov $\mathrm{V}$. S. in 1996 (Leonov, 1996) (due to replacement of the ether concept with the EQM concept) who named it quanton (recently, Leonov also raised an issue of returning the zero element to the Mendeleev's periodic table (Leonov, 2017c)).

The theory of Superunification (Leonov, 2010a) considers the process of Einstein's space-time quantization. Quantization is an energy process related to space filling with quantons. Quanton is not an elementary particle. It has a complex structure which includes four whole quarks: two electric $(+1 e$ and $-1 e)$ and two magnetic $(+1 \mathrm{~g}$ and $-1 \mathrm{~g})$ quarks forming a tetrahedron with two orthogonal force axes (dipoles) electric and magnetic. In general, two dipoles form an electromagnetic quanton quadrupole. Four mentioned quarks, that make up a quanton, combine electricity and magnetism in the form of a unified electromagnetic substance, the carrier of which is four-dimensional quantized space-time (QST) (Figure 1 (Leonov, 2013)).

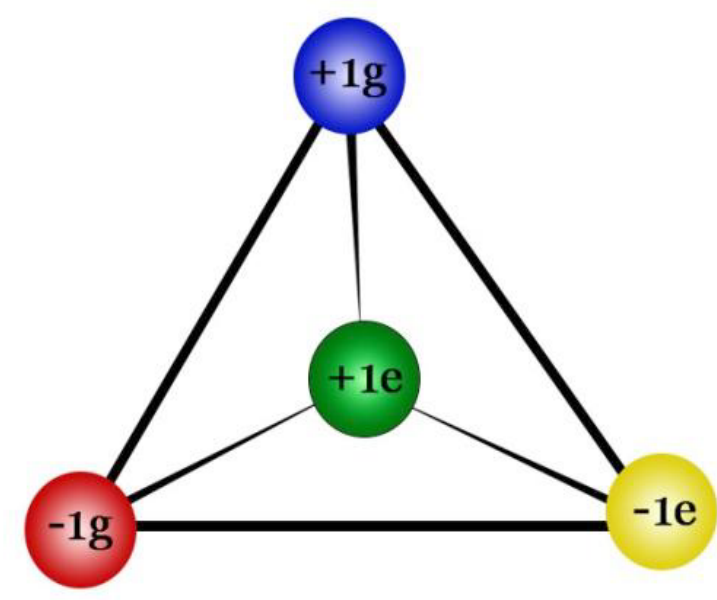

Figure 1. Quanton tetrahedron

The global vacuum (including all material insertions) is densely filled with mobile quantons representing a "boiling bouillon".

Quantons interact (attract and repulse) continuously due to their proximity, charge sign in adjacent quarks of neighboring quantons and orientation of dipole axes (see Figure 2 (Shkrudnev, 2017).

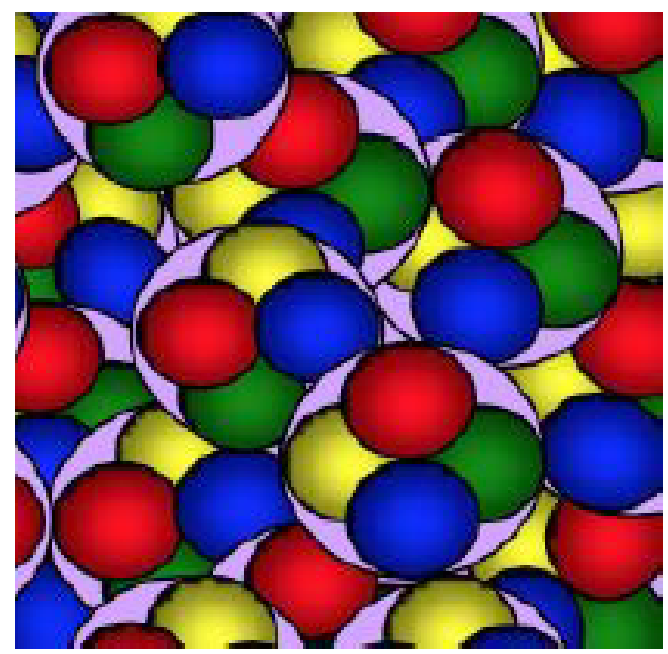

Figure 2. Quanton "broth" — quantized space-time 
The QST in the equilibrium state is an electromagnetic static field which is a carrier of super-strong electromagnetic interaction (SEI) - the fifth fundamental force (which Einstein and Davies were trying to find). SEl is that unified field combining gravity and electromagnetism, mentioned by Einstein in his general theory of relativity (GTR) (however, he failed to combine those). As a carrier of SEI, the QST possesses great energy density of approximately $10^{73} \mathrm{~J} / \mathrm{m}^{3}$ (Leonov, 2013).

The equilibrium state of a QST fragment implies that resultant vectors of axial forces of quark dipoles are zero in any direction (zero vectors). Introduction of a material object (containing a lot of free quarks) into the fragment perturbs the electromagnetic field, bending it relative to the initial equilibrium state (the resultant force zero vector acquires magnitude and direction). Control of free quarks' introduction allows affecting changes in the vector.

The discovery of the quanton in the form of a fourdimensional particle of a space-time quantum made it possible to give the GTR a quantum character. It also allowed V. S. Leonov to develop a quantum theory of gravity proceeding from the Einstein's concept of curved four-dimensional space-time as the basis of gravity.

It should be noted that the physical vacuum, penetrated by the elastic quantized medium, is subject to wave perturbations:

1) electromagnetic waves caused by the transverse electromagnetic polarization of quantons in space; 2) gravitational waves caused by the longitudinal deformation of the EQM; 3) torsional vibrations caused by the rotation of electromagnetic superstrings in the EQM (Leonov, 1997).

Leonov experimentally ascertained a ponderomotive (power conversion) interaction between electromagnetism and gravity. Actual anti-gravity effects were found. Experimental studies showed that external fields can interact with the EQM structure, resulting in stable antigravity effects (Leonov, 1997).

We can consider the outer space as an elastic super energy-dense medium having an electromagnetic structure with overall support and sufficient energy. All that is left for us to do is to learn how to interact with the environment and manage this interaction (Leonov, 1997).

As it is stated in the theory of Superunification, all known types of energy (chemical, nuclear, electromagnetic, gravitational, mechanical, etc.) imply extraction of SEI global energy and its further transformation allowing opening up a new direction in energetics - quantum energetics (Leonov, 2010a).

\section{Quantum engine framework}

The operation principle of quantum engines is based on the interaction of the field of a rotating electromagnetic quadrupole with the quantized medium (vacuum field). The quantum engine operation is based on the quantized space-time curvature (deformation) effect. As a result of this interaction, thrust appears, which affects movement in space (Leonov, 1997).
In the EQM theory, the Lorentz force (Repchenko, 2017) (which is directed towards the region of minimum magnetic field strength) is of great importance. Its character is associated with space deformation in a local region. This deformation can be created artificially as in a current circuit. The effect of the Lorentz force during rotation of an electromagnetic quadrupole is of particular interest as the quadrupole experiences axial thrust during rotation. The quadrupole is as though screwed into the medium, pushed away from it and, as a result, moves forward like an air propeller (Leonov, 1996). The quadrupole thrust value is determined by the charge $\mathrm{e}(\mathrm{g})$, rotation frequency and coefficient KR (which manifests at relativistic velocities).

Thus, if a quanton with its structure of the electromagnetic quadrupole is forced to rotate, then field thrust appears, which can cause it to move in space (together with the structural enclosure of the working chamber in a quantum engine) at a high speed.

Therefore, physics of the process is based on the interaction of fields (the rotating field of the electromagnetic quadrupole and the sign-alternating field of the quantized medium). In other words, quantum engines are engines, thrust of which is caused by the interaction of fields.

In a quadrupole cell, the quadrupole is held and rotates without energy emission beyond the structural enclosure. It is a structural property of quantum engines. Based on this property, in quantum engines, almost $100 \%$ of energy are converted into motion. In conventional chemical-fuel engines, such conversion cannot be obtained: their conversion efficiency is extremely low (Leonov, 1996).

According to Newton's third law, for every action there is an equal reaction and thrust is created due to repulsion from another mass or body. As for car wheels, they repulse from the road surface. In the jet engine, thrust is created due to the ejection of reactive mass, as if repulsing from this mass. Air and hydraulic propellers, screwing into the air or hydraulic medium, reject the mass of such medium, repulsing from it.

But is it possible to repulse from vacuum? The theory of Superunification states that it is possible if we consider the space vacuum as an elastic quantized medium (quantized space-time (QST) of primary nature) from which it is possible to repulse (Leonov, 2010a).

The theory of Superunification also states that weightless quantized space-time penetrates all weighable bodies. In this case, all weighable bodies represent an integral part of weightless quantized space-time. The body mass is formed as a result of the spherical deformation (bending according to Einstein) of weightless quantized space-time by elementary particles making up the body. In this case, the body mass represents an integral part of the elastic quantized medium, its energy cluster. The mass, as a gravitational charge, is a secondary formation in quantized spacetime (Leonov, 2010a). 
All known methods of producing thrust are based on the external action with the repulsion from the known media. In this case all known apparatuses for thrust generation shall be treated as closed quantum-mechanical systems. In accordance with the theory of Superunification, the quantum engine is an open quantum-mechanical system where thrust is created inside the body of the operating unit (activator) of the quantum engine.

To create thrust without the ejection of reactive mass it is necessary to switch over to the open quantummechanical systems, treating quantized space-time as an elastic quantized medium. Consequently, it is possible "to push away" from such an elastic quantized medium thus generating thrust. In this case, there are no contradictions with Newton's third law, whose fundamentality was thoroughly checked and completely confirmed by the theory of Superunification (Leonov, 2010a, 2010b).

The very process of creating the thrust inside the operating unit of the quantum engine is connected with Einstein's "bending" of quantized space-time. Based on the theory of Superunification, the Einstein's bending of space-time looks like a real deformation of the elastic quantized medium inside the operating unit of the quantum engine (Leonov, 2010a).

This deformation redistributes the quantum density of the medium inside the body of the operating unit of the quantum engine. This leads to the appearance of gradient thrust forces inside the operating unit. Thus, for the first time gravity and inertia are controlled. This once again confirms the fundamentality of the Einstein's theory that gravity is based on bending of quantized space-time (Leonov, 2010b).

The deformation of QST in the local region inside the operating unit (activator) of the quantum engine corresponds to the energy which is spent by the body on its acceleration. In this case, we have the classical law of energy conservation. Deformation of vacuum takes place in the body of the operating unit of the quantum engine which actively interacts with the vacuum medium which penetrates the body. The internal thrust force appears inside the body of the operating unit. This is not external repulsion as in the jet engine, it is internal repulsion. If the classical theory forbids motion without the ejection of reactive mass, the theory of Superunification permits this motion (Leonov, 2010a).

Thus, quantum engines are engines, thrust of which is caused by the interaction of fields (Leonov, 1996).

It should be noted that, during motion with acceleration, particular regimes form inside QST when energy recovery is observed during deceleration, therefore, the consumed energy returns and can be used again. Such regimes are used in hybrid circuits of automobiles with electric transmission or in electric trains. In other words, inertia has a capacity for energy recovery.

Inertia regimes with energy recovery can be actively used in quantum engines. The capacity of quantum engines for energy recovery during thrust generation provides economical power cycles in quantum engines.
It is necessary to compensate energy losses due to friction in engine mechanisms and ohmic losses in electrical wires and windings of activators (Leonov, 2010b).

The efficiency of the quantum engine during thrust generation can exceed that of conventional engines 20 or more times. However, to activate SEl energy in the quantum engine, additional power is required which would compensate heat losses in wires and bearings. However, those losses are within 5\% (Leonov, 2010b).

\section{Quantum engines: prototypes of engineering designs and development models of machines with quantum engines}

Prototypes of quantum (field, vacuum) engines and energy sources were described in works of $\mathrm{N}$. Tesla, S. Floyd, T. Brown, V. Shchabetnik, V. Leonov, V. Prokopyev, B. Ignatov, Yu. Ivanov, A. Miteshov and other inventors (Miteshov, 2015).

The Leonov's quantum engine is very promising as it has at least two laboratory models (Leonov, 2009, 2010b, Petrov, 2015). The concept, method and design of the quantum engine are protected by patent No. 2185526 (Russian Federation, priority date: 21.05.2001) "A method of thrust generation in vacuum and a field engine for spaceships (options)" (Leonov, 2002).

The technical result of the quantum engine (Leonov, 2002) is achieved by the following: during thrust generation in vacuum, the operating unit is exposed to a system of rotating crossing heterogeneous electric and magnetic fields, and, simultaneously, electrical and magnetic properties are set for such operating unit. In these circumstances, the quantum density of the vacuum field environment is redistributed inside the operating unit in a direction opposite to the thrust vector caused by the deformation of the vacuum field.

Meanwhile, the operating unit rotates. The thrust vector is divided into two vectors (normal and tangential). The normal vector allows producing thrust. The tangential vector allows producing torque providing power to supply the system of crossing heterogeneous electric and magnetic fields and system of their rotation.

Despite the fact that this patent has rather simple description for the design of operating units (activators) and operation principle of the quantum engine, it took the author years to create an operative structure. With a unit mass of $50 \mathrm{~kg}$ (including the chassis), he managed to achieve a thrust of $0.1 \mathrm{~N}$, and in 2009 this value increased to $500 \mathrm{~N}$ (Leonov, 2009).

In 2014, a prototype quantum engine (1 kW) produced a thrust of $5,000 \mathrm{~N}$ in pulse (for comparison, a modern $1 \mathrm{~kW}$ jet engine produces a thrust of only 1 N) (Petrov, 2015).

According to Leonov V. S., there are no restrictions regarding thrust of quantum engines. He developed a method for calculating the design parameters of the quantum engine for any thrust, including $1,000 \mathrm{~N}$, 10,000 N and more (Leonov, 2010a). 


\section{Conceptual schemes and advantages of cars with quantum engines, pointed out by V. S. Leonov}

The main direction of laboratory researches on quantum energy is related to the development of quantum engine concepts for field space exploration. It also noted that air transport with field engines will be scarcely different from space transport.

Meanwhile, above-ground motor transport can undergo serious transformation.

Diagrams of quantum engine installation in cars are presented in Figure 3 (Leonov, 1997). Propulsive engine 2 is installed in the rear of the car (a). Motion is caused by propulsive field thrust $R$. It is reasonable to install engines generating horizontal and vertical thrust in high-speed cars (b).

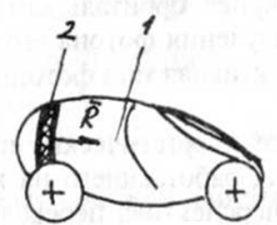

a)

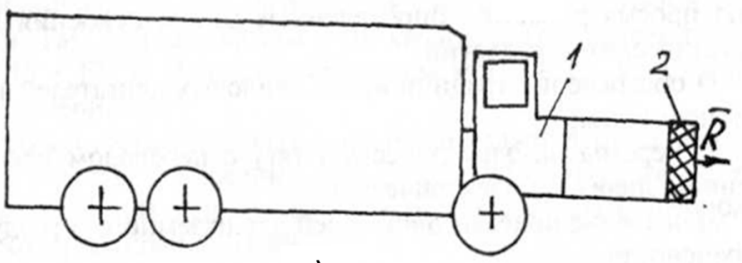

c)

Figure 3. Diagrams of quantum engine installation in cars (Leonov, 1997): 1 - frame of a car; 2 - quantum engine

In this case, the load on wheels decreases. It is possible to install the engine at the front of the car (c), ensuring motion caused by thrust $R$.

A distinctive feature of cars with quantum engines is transmission removal. It allows for the installation of free suspension bracket of wheels. The car design includes the following components: a body, a quantum engine, and controlled wheels.

Despite the simple car design, implementation of quantum engines will require new cutting-edge technologies. Production of quantum engines can be expensive, therefore, such cars will be more expensive than those used nowadays (Leonov, 1997).

In Figure 4, a scheme of an off-road on an antigravity cushion, capable to overcome water obstacles, is presented (Leonov, 2010a). This off-roader will be able to move as a flying car at a certain height from the water surface.

Altitude control (when flying over obstacles) will be provided by an automation system controlled by radar scanner 3. The radar scanner will ensure safe movement of the car, preventing collisions. The radar-scanner with microprocessor control is capable to control car movement in the autopilot mode.

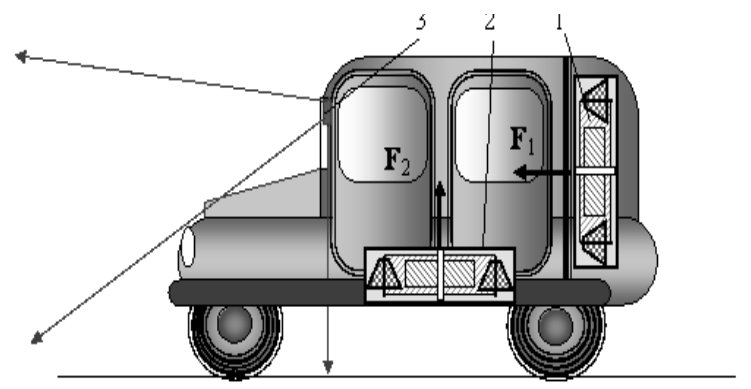

Figure 4. An off-road vehicle on an anti-gravity cushion with two quantum engines 1 and 2 - with horizontal $F_{1}$ and vertical $F_{2}$ thrust and radar scanner 3

The main advantages of the quantum engine compared to conventional engines, pointed out by Leonov, are as follows (Leonov, 2010a):

1) no chemical fuel is required for quantum engine operation;

2) unlimited movement distance without refueling;

3) significant reduction in material costs for traffic infrastructure (filling stations, power transmission lines for city transport, etc.);

4) high environmental friendliness and safety;

5) The quantum engine allows starting the car with fixed thrust, making maximum use of its power, avoiding losses inherent in ICE under unstable conditions (ICE lose $80 \%$ power due to low efficiency at low engine speed during starting, $50 \%$ power - during clutching; as a result, ICE use only about $10 \%$ power at the start of the car).

Design features of cars with quantum engines and differences between such cars and cars used nowadays

Let us try to predict possible design and operating features of the car with a quantum engine in detail (compared to modern cars with an ICE or electric engine), from the point of view of an expert-automobilist.

Obviously, we cannot perform an adequate analysis of all car properties (due to the lack of any data as designs of cars with quantum engines do not exist yet).

However, according to the author's opinion, their review can be useful for developers of future concept designs:

1. The loading pattern will change: the suspension can become less elastic, and the body - more durable. Earlier, the total propulsive force was used to propel both sprung and unsprung weights. In quantum engines, the propulsive/pulling force is used to propel the sprung weight with a rear-/front-positioned quantum engine, but the propulsive force is always applied only to the sprung weight.

2. If the body of the modern car is designed in such a way that the front and rear parts of the load-bearing 
structure are easily deformed during collision, then, in cars with quantum engines, the frame and body shall be rigid and reliable (at least in components transmitting traction).

3. The point of traction application is located above wheel axles (particularly, above point of tire contact) which can change the movement mechanics.

4. Lack of longitudinal and transverse reaction overturning moments in cars with quantum engines.

5. In case of a front-mounted quantum engine (see Figure 3c), this propulsion unit becomes a rigid anchoring point for the "towed" car, which would result in increased angular vibrations of the car (with two degrees of freedom) around this point. It will adversely affect the car stability, dimensions of the dynamic movement corridor, vibration load of the structure, cargo safety and comfort of passengers.

6. Cars with quantum engines do not require a main power drive: gearbox, drive shafts, a main reduction gear, and pivot joints for front-wheel drive cars.

7. No inter-wheel and inter-axle differentials, or freerunning differentials (for special machines) are required.

8. Absence of drive shafts, final drive housings and differentials makes it possible to arrange a clad floor, which improves the cross-country ability, reducing whirling and air resistance.

9. The possibility of implementing significant accelerations (more than 1g), both negative and positive (the maximum will be restricted by biomechanical limits of passengers and drivers).

10. Automatic energy recovery during cyclic movement.

11. Traction orientation along the longitudinal axle (especially in case of a front-mounted quantum engine, see Figure 3c) will lead to insufficient cornering ability due to large front slip angles (it is a disadvantage compared to modern front-wheel drive cars). This will require a turned vector of traction generated by the engine.

12. A controlled vertical (anti-gravity) component of traction will allow "suspending" the vehicle, improving its off-road mobility.

13. It is possible to use two quantum engines in allterrain vehicles: for horizontal and vertical traction (see Figure 4).

14. According to the author's opinion, it is possible to install one quantum engine with the transverse axis of turning (or with hinge mounting to the body) to regulate the magnitude and direction of the traction vector. It will improve controllability and off-road performance.

15. This advantage of hinge mounting allows using steering wheel handle, which will improve ergonomics, controllability, cornering ability and off-road performance.

16. Essentially, it means transition to the "auto-avia-craft".

17. Better cross-country ability in comparison with modern cars.

18. No wheel slippage (irrespective of roads and longitudinal forces).

19. A vertical vector can put pressure on wheels on wet roads and in other conditions of insufficient adhesion.
20. Cars with quantum engines can climb steep hills (even without high-speed dynamics).

21. The danger of rollover is eliminated/reduced when the transverse force vector is used by an automatic control system and/or the connection with the steering wheel handle. Cars with quantum engines will be able to move over steep hillsides.

22. Wheels turn around the vertical axis more easily and simultaneously on all axles. It facilitates maneuvering.

23. Cars with quantum engines can still require an independent drive for wheels to facilitate maneuvering. In this case, it will be easier to implement the connection with the parking radar compared to such connection in modern cars.

24. Automatic cruise control will make driving easier (no transmission is required (neither mechanical nor automatic); no servo-motor drive for throttle or engine injector is required; no partial braking is required).

25. The emergency braking system will be more effective: a vertical force vector will put pressure on wheels to maintain roadholding during corner braking, with optimization of weight distribution of vector components by axles and wheels.

26. Complex downhill assist control systems (with use of the main brake system, engine brake and ABS) will be unnecessary. During motion of the car with a quantum engine, the traction vector shall be reversed and its value shall be adjusted (wheels will not slip). The downhill assist control system (if any) will be simpler.

27. Noise level: wheels used only in the driven mode will make less noise, and the engine, hopefully, will too.

28. No vibration (therefore, noise) from elements of the power drive.

29. It is still unclear how the quantum engine will be cooled, what thermal impact on a driver and passengers will be. It is also unclear if thermal protection is required.

30. Atmospheric conditions will not affect the operation of the quantum engine.

31. Less chemical emissions. The level and biological effect of high-frequency oscillations in quantum engines are still unclear.

32. Less tire wear.

33. No need for regular refueling.

34. The entire system of car maintenance will change.

35. Changes in roads and traffic regulations for cars with quantum engines will be observed as well.

\section{Conclusion}

Despite the lack of global experience in development of cars with quantum engines and their mass-production models, it was possible to review aspects of their development and operation.

The judgmental forecast allowed distinguishing a number of certain advantages of cars with quantum engines (compared to modern cars): simplified design; higher level of acceleration and braking 
dynamics, stability, controllability, chemical environmental compatibility, noise compatibility; no need for regular refueling, automatic energy recovery, less tire wear, etc.

It is still unclear what noise and high-frequency characteristics will quantum engines have, what influence of local deformation of the gravity field on the human body and other innovations will be. Properties with significant changes (possibly, in the undesirable direction) were also noted; they will require new calculation methods, research methods and solutions, e.g. regarding loading patterns.

The author's opinion is that the oscillatory systems shall be reviewed as well.

Obviously, mass production cars with quantum engines will lead to significant changes in the entire infrastructure and transportation technology. 


\section{References}

Davies, P. (1985). Superforce. New York: Touchstone.

Kotikov, Ju., Lozhkin, V.N. (2006). Transportnaia energetika [Transport energetics]. Moscow: Publishing Center "Academia", p.272. (in Russian)

Leonov, V.S. (1996). Teoriia uprugoi kvantovannoi sredy [Theory of elastic quantized medium]. Minsk: Bisprint, p.156. (in Russian)

Leonov, V.S. (1997). Teoriia uprugoi kvantovannoi sredy. Chast 2 . Novye istochniki energii [Theory of elastic quantized medium. Part 2. New energy sources]. Minsk: Polibig, p.122. (in Russian)

Leonov, V.S. (2002). Patent No. 2185526 (Russian Federation). Sposob sozdaniia tiagi v vakuume i polevoi dvigatel dlia kosmicheskogo korablia (varianty) [A method of thrust generation in vacuum and a field engine for spaceship (options)]. Bulletin No. 20 dd. 20.07.2002 (priority date: 21.05.2001). (in Russian)

Leonov, V.S. (2009). Rezultaty ispytanii 2009 goda kvantovogo dvigatelia dlia sozdaniia tiagi bez vybrosa re-aktivnoi massy [Quantum engine test results regarding thrust generation without ejection of reactive mass (2009)]. Available at: http://www.rnto. club/up/files/321/rezultati_ispitaniy_2009.pdf (accessed on: 28.01.2018) (in Russian)

Leonov, V.S. (2010). Quantum Energetics. Volume 1. Theory of Superunification. Cambridge International Science Publishing, p.745.

Leonov, V.S. (2010b). Kosmos: rezultaty ispytanii kvantovogo dvigatelia dlia sozdaniia tiagi bez vybrosa [Space: quantum engine test results regarding thrust generation without ejection]. Available at: http://www.astronomy.ru/forum/index.php/ topic,77730.0.html (accessed on: 28.01.2018) (in Russian)

Leonov, V.S. (2013). Einstein vs Higgs: or what is the mass? Available at: http://www.trinitas.ru/eng/doc/0016/001e/3139-In.pdf (accessed on: 28.01.2018)

Leonov, V.S. (2016). Kosmicheskii minikorabl s kvantovym dvigatelem doletit do Alfy Tcentavra za 8 let [Mini spaceship with a quantum engine will reach Alpha Centauri in 8 years]. Available at: http://leonovpublitzistika.blogspot.ru/2016/04/8.html (accessed on: 28.01.2018) (in Russian)

Leonov, V.S. (2017a). O nauchno-tekhnicheskoi revoliutcii [On the scientific and technological revolution]. Available at: http:// www.quanton.ru/o-nauchno-tehnicheskoj-revoljucii/ (accessed on: 28.01.2018) (in Russian)

Leonov, V.S. (2017b). Novye energeticheskie kontceptcii [New energy concepts]. Available at: http://www.quanton.ru/novyejenergeticheskie-koncepcii/ (accessed on: 28.01.2018) (in Russian)

Leonov, V.S. (2017c). Otkrytie nulevogo elementa tablitcy [Discovery of the zero element in the Mendeleev's periodic table]. Available at: http://zg5.cosmotest.ru/leonov7.php (accessed on: 28.01.2018) (in Russian)

Leonov V.S. (2017d) Kvantovye dvigateli-generatory [Quantum engine-generators]. Available at: http://www.quanton.ru/ kvantovye-dvigateli-generatory/ (accessed on: 28.01.2018) (in Russian)

Mendeleev, D.I. (1905). Popytka khimicheskogo ponimaniia mirovogo efira [An attempt towards a chemical conception of the ether]. Saint Petersburg: M. P. Frolova's tipolithography. (in Russian)

Miteshov, A.A. (2015). Vakuumnyi dvigatel - budushchee kosmonavtiki [Vacuum engine is the future of space exploration]. Available at: http://www.avmodels.ru/news/interesting/index_full.html?id=4333 (accessed on: 28.01.2018) (in Russian)

Nikitin, A.P. (2014). K energodinamike dvizhushchegosia kosmosa [To the energy dynamics of moving space]. Zhurnal russkoi fizicheskoi mysli [Journal of Russian Physical Thought], 1-12, pp. 103-118. (in Russian)

Parker, B. (1991). Mechta Einshteina. V poiskakh edinoi teorii stroeniia Vselennoi [Einstein's dream. The search for a unified theory of the Universe] (translated from English by V. I. and O. I. Matsarskikh). Moscow: Nauka, p.223. (in Russian)

Pauli, W. (1975). Fizicheskie ocherki [Physical essays]. Moscow: Nauka. (in Russian)

Petrov, A. (2015). Rossiia uspeshno ispytala antigravitatcionnyi dvigatel Leonova [Russia successfully tested the Leonov anti-gravity engine]. http://www.km.ru/science-tech/2015/01/16/nauka-i-tekhnologii/753573-rossiya-uspeshno-ispytalaantigravitatsionnyi-dvi (accessed on: 28.01.2018) (in Russian)

Puthoff, H. (2010). Advanced Space Propulsion Based on Vacuum (Spacetime Metric) Engineering. JBIS, 63, pp. 82-89.

Repchenko, O. (2017). Sila Lorentca v Polevoi fizike [Lorentz force in field physics]. Available at: http://www.fieldphysics.ru/ lorentz_force/ (accessed on: 28.01.2018) (in Russian)

Shkrudnev, F.D. (2017). Vselennaia: kipiashchii "bulon" iz kvantonov [The Universe: a boiling "bouillon" of quantons]. Available at: https://shkrudnev.com/index.php/home/drugie-avtory/v-s-leonov/item/1866-vselennaya-kipyashchij-bulon-iz-kvantonovbulon-iz-kvantonov (accessed on: 28.01.2018) (in Russian)

Veinik A.I. (1991). Termodinamika realnykh protcessov [Thermodynamics of real processes]. Minsk: Nauka i Tekhnika, p.576. (in Russian) 